ISSN electrónico: 2602-8069

\title{
LA METÁFORA, RECURSO PEDAGÓGICO EN ESPACIOS DE EDUCACIÓN NO FORMAL MEDIADOS POR EL USO DE TIC: UNA EXPERIENCIA ECUATORIANA
}

Metaphor, a pedagogical resource in non-formal educational
spaces mediated by the use of ICT: an Ecuadorian experience

Luis Fernando Vallejos

Fundación Investigación Psicología y Comunidad (IPC)

luisfernandovallejos.ipc@gmail.com

Verónica Egas-Reyes

Pontificia Universidad Católica del Ecuador

MEGAS776@puce.edu.ec

Diego Ordóñez-Camacho

Universidad Tecnológica Equinoccial

dordonez@ute.edu.ec

Fecha de recepción del artículo: 26/03(2018

Fecha de aceptación definitiva: 18/05/2018 
Luis Fernando Vallejos - Verónica Egas-Reyes - Diego Ordóñez-Camacho

La metáfora, recurso pedagógico en espacios de educación no formal mediados

por el uso de TIC: una experiencia ecuatoriana

\section{RESUMEN}

A continuación, se expone una experiencia socioeducativa que hace uso de la metáfora como herramienta pedagógica para la significación del uso de recursos tecnológicos, en el desarrollo de competencias para la vida, aplicada en espacios de educación no formal. El método consistió en la construcción de microproyectos insertos en módulos temáticos y talleres recreativos acordes al entorno comunitario y al universo simbólico de los participantes, para facilitar la abstracción de información técnica referida a la robótica y programación básica, así como también, actividades que aportan al proceso de autoconocimiento de los participantes. La propuesta plantea un esquema de trabajo cuyo principal mecanismo pedagógico es la metáfora. Este tropo permite la incorporación de la robótica y su consecuente asociación con elementos inherentes al desarrollo de cuatro competencias para la vida (liderazgo, responsabilidad, creatividad e innovación), en grupos infantoadolescentes que viven en condición de vulnerabilidad en sectores rurales y urbano-marginales del Ecuador. Como resultado de la experiencia se disminuye la brecha digital en grupos prioritarios. El uso de la tecnología rebasa lo instrumental y se convierte en una herramienta simbólica para estimular la curiosidad y para la producción de sentido a través de la cual los participantes se proyectan en un plan de vida acorde a su deseo.

Palabras clave: infantoadolescente, aprendizaje por metáfora, TIC, educación no formal, trabajo comunitario

\section{ABSTRACT}

This paper presents a sociological-educational experience, using metaphor as a pedagogical tool that provides meaning to technological resources, when employed to develop life-long skills on non-formal educational spaces. The method consisted on building micro-projects, inside thematic clusters and recreational workshops, adapted to the community environment and symbolic universe of the participants. This allowed for the technical data abstraction regarding basic robotic and programming, and for the execution of activities that improved the participants' self-knowledge. Metaphor, proposed as the main pedagogical tool, allowed to intrinsically associate robotic' technical elements, with four life-long skills development (leadership, responsibility, creativity and innovation), on vulnerable children-teenager groups, living on impoverished and underdeveloped Ecuadorian urban, suburban and rural regions. As a result of this experience, the digital breach for these groups of participants is reduced, and technology goes beyond the mere devices and instruments, becoming a symbolic tool that stimulates curiosity and provides a deeper meaning that the participants use to project themselves into a life plan fitting their own desires and aspirations.

Keywords: children, teenagers, learning through metaphor, ICT, non-formal education, community work. 
Luis Fernando Vallejos - Verónica Egas-Reyes - Diego Ordóñez-Camacho La metáfora, recurso pedagógico en espacios de educación no formal mediados por el uso de TIC: una experiencia ecuatoriana

\section{INTRODUCCIÓN}

La lucha social por la igualdad de oportunidades en el Ecuador ha conducido a organismos públicos y privados a plantear espacios educativos distintos a los tradicionales para que grupos vulnerables superen su adversidad (Bunch, 2016; Vega, 2016). Estas acciones, en su conjunto, pretenden la restitución de derechos de grupos prioritarios (Observatorio Social del Ecuador, 2004). Estas propuestas se inscriben en la llamada educación no formal o educación fuera del aula (Grasso, Pagola y Zanotti, 2017). Son iniciativas desarrolladas por técnicos sociales dirigidas principalmente a niños, niñas y adolescentes, cuyo objetivo es prepararlos actitudinalmente por medio de la coconstrucción de aprendizajes prácticos que resuelvan los retos de la vida (Perrenoud, 2012). Acompañar al adolescente en su pasaje de la vida infantil a una vida adulta, implica abrir un espacio dialógico para tratar las interrogantes propias de la reconstrucción de su identidad (Aberastury y Knobel, 2004). Este ejercicio no es sencillo y demanda la incorporación de recursos novedosos que den paso a la circulación de la palabra. En consecuencia, este tipo de educación propone el uso de material didáctico, lúdico y ahora tecnológico, que dinamiza el intercambio reflexivo en los participantes permitiendo generar una proyección de vida acorde a su deseo y anhelos (Garganté y Majós, 2006). En esta medida se plantean marcos pedagógicos que dan sentido al uso tecnológico (Lion, 2006).

Las tecnologías de la información y comunicación (TIC) han influido en todas las esferas de la vida humana, y han transformado los procesos de intercambio entre el individuo y su entorno físico y social; los espacios de enseñanza-aprendizaje no escapan de su alcance (Castells y Andrade, 2010). La incorporación de recursos tecnológicos, como la robótica y programación, en el ámbito de la educación no formal, están iniciando y apuntan a lograr en el adolescente una significación de sí mismo como hacedor de su futuro y agente social activo de su comunidad (Sierra Caballero, 2013). Se propone, entonces, una estructura pedagógica basada en el aprendizaje por metáfora (Pochulú, Abrate, y Visokolskis, 2011), que permita la asociación de contenidos técnicos básicos de robótica y programación con aspectos de la vida psicosocial de los participantes, y el fortalecimiento de las llamadas competencias para la vida.

En el 2005, el Parlamento Europeo (a través de la Cumbre de Ministros de Educación de la OCDE-París) propuso el concepto de competencias como una combinación de habilidades prácticas y cognitivas interrelacionadas entre sí, en el que se incluye a las emociones, la conducta, el comportamiento y otros aspectos sociales articulados de manera simultánea para crear un contexto que permita a los ciudadanos tener la capacidad de aportar a la sociedad a través del aprendizaje, el cual se enmarca en un proceso formativo y en elementos que permiten un aprendizaje autónomo a largo plazo (lifelong learning). Según Romeroy Turpo (2015), las TIC se articulan directamente con las competencias del siglo XXI debido a que: "...la tecnología no solo es un argumento de los "referenciales de competencias" (frameworks), sino que responden a las nuevas necesidades, es decir, a promover un conjunto de competencias acerca de cómo utilizar de forma eficaz al gestionar, evaluar y producir información" (p. 6).

\section{Aprendizaje en entornos educativos no formales}

Entendemos el aprendizaje, a nivel humano, como un proceso sostenido por el uso del lenguaje para significar los acontecimientos a los que se expone un individuo, y con los cuales determina intencionalidades para generar conductas que modifiquen su entorno. Es un proceso dinámico que evoluciona con el tiempo y en el que actúan, de modo recíproco, mecanismos psicológicos y circunstancias del medio ambiente 
Luis Fernando Vallejos - Verónica Egas-Reyes - Diego Ordóñez-Camacho La metáfora, recurso pedagógico en espacios de educación no formal mediados por el uso de TIC: una experiencia ecuatoriana

(Castro Pérez y Morales Ramírez, 2015; Esteve, 2016; Palma, Agudelo y Aguilar, 2017; Zapata-Ros, 2015).

Todos los escenarios de la vida proveen aprendizajes si se sabe generar sentido de una experiencia. Cabe mencionar tres espacios educativos que actúan complementariamente. El primero se refiere a los espacios de educación formal o dentro del aula, en el cual el conocimiento proviene de asignaturas encaminadas hacia la profesionalización de los sujetos, saberes escolares ajustados a una demanda laboral global (Medina, 2015). El segundo espacio de enseñanza corresponde a la educación informal, nutrida por la experiencia de las figuras significativas del círculo familiar o social que advierten, a modo experiencial, lecciones que pueden ser útiles para afrontar ciertas contingencias de la vida (Costa, Cuzzocrea, y Nuzzaci, 2014; Martínez Hernández, Nolla Cao, Vidal Ledo y De la Torre Navarro, 2016; Pessoa, Serrano y Rodríguez, 2015). Tercero, tenemos a la educación no formal o fuera del aula, que plantea entornos de aprendizaje fundados en el intercambio reflexivo entre pares quienes son guiados por un acompañante -adulto- que dinamiza la producción de conocimiento en la práctica de ejercicios recreativos, artísticos o deportivos (Ramírez, 2017; Suárez y Santana, 2016; Valenzuela, 2016). Estas actividades son generalmente la puerta de entrada para el abordaje de problemas cotidianos. Dicho conocimiento no se define en términos disciplinares, sino que son derivaciones amplias del ejercicio comunicativo (Ruiz y López, 2017).

Entonces, el aprendizaje obtenido en entornos educativos no formales proviene de la vivencia de una práctica social determinada, su intención es que el sujeto pueda combinar sus recursos intelectuales y emocionales para hacer uso máximo de sus habilidades al momento de enfrentar una contingencia; es decir, son aprendizajes para la vida. En este sentido, el aprendizaje existe para adquirir dominio y responsabilidad en situaciones concretas, movilizar actitudes y saberes hacia un propósito, producir nuevos aprendizajes y recursos para aplicarlos según la necesidad y finalmente para canalizar el potencial individual hacia una acción colectiva (Correa, 2017). Es bajo esta lógica que aparece el concepto de aprendizajes invisibles, el cual es una propuesta actual y se encuentra en constante construcción.

El aprendizaje invisible es una propuesta conceptual que surge como resultado de varios años de investigación y que procura integrar diversos enfoques en relación con un nuevo paradigma de aprendizaje y desarrollo del capital humano, especialmente relevante en el marco del siglo XXI. Este enfoque toma en cuenta el impacto de los avances tecnológicos y las transformaciones de la educación formal, no formal e informal, además de aquellos metaespacios intermedios. Bajo este enfoque se busca explorar un panorama de opciones para la creación de futuros relevantes para la educación actual. Aprendizaje invisible no pretende proponer una teoría como tal, sino una metateoría capaz de integrar diferentes ideas y perspectivas. Por ello ha sido descrito como un protoparadigma que se encuentra en fase beta y en plena etapa de construcción (Cobo y Moravec, 2011, p. 68).

Construir una metodología para una educación no formal demanda el uso de estrategias pedagógicas que expongan a los participantes ante problemas reales, dispuestos en simulaciones, investigaciones. Experimentaciones $u$ otros acontecimientos en los cuales se ilustren las limitaciones, fortalezas o debilidades para el trabajo participativo. Por tanto, el aprendizaje no se determina a partir de saberes predeterminados como en la educación formal o informal, sino son inferidas de una actividad social. Partimos de la noción que el sujeto se construye de las actividades y de las tecnologías que median sus relaciones. 
Luis Fernando Vallejos - Verónica Egas-Reyes - Diego Ordóñez-Camacho La metáfora, recurso pedagógico en espacios de educación no formal mediados por el uso de TIC: una experiencia ecuatoriana

\section{La tecnología como vía para la producción subjetiva}

Si entendemos a las tecnologías como un recurso utilizado por el hombre para ejercer cierto control del ambiente y transformar su entorno próximo, podríamos afirmar que las mismas existen y han evolucionado a la par del desarrollo humano, forman parte de su historia y se conectan en una relación de mutua afectación. La importancia de la tecnología ha variado a lo largo del tiempo, haciendo evidente que su sentido en cuanto a uso y apropiación dependerá de contextos específicos de significación.

Lion (2006) diferencia al menos tres tipos de discurso que dan cuenta de la relación entre los seres humanos y la tecnología. El discurso determinista-esencialista supone que la tecnología posee cierta autonomía, se deslinda del contexto social del cual emerge, por tanto, la persona es un agente ausente que es modificado unilateralmente por los avances tecnológicos. El discurso racional-eficientista por su parte, señala que la tecnología tiene sentido en la medida que contribuya con la optimización de recursos, el rendimiento y la eficacia de los sistemas productivos; en consecuencia, debe aminorar costos y tiempo. Finalmente, el discurso didácticotecnológico destaca una función educativa en el uso de la tecnología, refiere a las nuevas formas de producir sentido en espacios mediados tecnológicamente, situación que afecta directamente al qué y cómo enseñar (Lion, 2006). Aunque estas tres perspectivas marcan criterios distintos, lo reconocible es que las tecnologías son parte del ser y hacer humano. Siguiendo a Lion coincidimos cuando afirma:

Las tecnologías no nos sustituyen. No podrán hacerlo. Pero tampoco nosotros podríamos sustituirlas. Es una necesidad mutua en tanto formamos parte de una cultura con herramientas que nos transforman, transforman nuestro trabajo y nosotros mismo creamos y recreamos el marco de esta dialéctica (2006, p. 36).

El desarrollo evolutivo de los seres humanos les ha otorgado la capacidad de inventar prácticas y crear herramientas que les facilitan y organizan la vida. Este mismo crecimiento produce, además, cambios en la conducta, en la condición mental y social de la persona, que a la par de hacer uso de recursos materiales y técnicos, se vale de herramientas culturales, provistas en el lenguaje para recrear un universo imaginario con el cual se abstrae e interactúa con el mundo. Las tecnologías son como tal, artefacto cultural (Coll, 2008) productos testimoniales de la interacción del individuo con el ambiente. En el 2008, Martínez, Sádaba y Serrano realizaron una investicación con relación al desarrollo de las llamadas competencias digitales en la comunidad virtual ScolarTIC; dentro de las conclusiones se determinó que en una estructura dinámica y descentralizada de intercambio de conocimiento se pueden desarrollar diversas áreas de competencia digital: cómo navegar, buscar, evaluar, almacenar y recuperar información, tanto en teléfonos inteligentes como en tabletas y otros dispositivos.

Cuando se interacciona con un artefacto o sistema tecnológico, al mismo tiempo, el universo simbólico de la persona se afecta y viceversa, confirmando que existe una relación tangencial entre tecnología y subjetividad. Resultado de ello surgen nuevas prácticas sociales en ambientes socioeconómicos y socioculturales distintos (Belçaguy, Cimas, Cryan y Loureiro, 2015). En el campo de la subjetividad, aprendizaje y tecnologías, Dolors Reig (2012), psicóloga e investigadora sobre jóvenes y las Tic, propone el concepto de Tecnologías para el Empoderamiento y la Participación (TEPs), en el cual propone que las nuevas tecnologías, más allá de comunicar, crean tendencias y cambian el medio ambiente de los jóvenes, además de 
Luis Fernando Vallejos - Verónica Egas-Reyes - Diego Ordóñez-Camacho La metáfora, recurso pedagógico en espacios de educación no formal mediados por el uso de TIC: una experiencia ecuatoriana

aportar en su autodeterminación y el fortalecimiento de los valores personales, para que, de esta manera, puedan tener incidencia social y autorrealización personal.

\section{Robótica como artefacto mediador para la educación no formal}

La robótica es una ciencia transdisciplinaria que integra conocimientos de la informática, electrónica, mecánica, ingeniería de control e inteligencia artificial, para el diseño y construcción de artefactos con comportamiento autónomo previamente regulados. Forma parte de la familia de las TIC en cuanto ha hecho posible la reconfiguración de los entornos de relación entre las personas y sus ambientes. La robótica ha sido utilizada en entornos de aprendizaje formal desde hace varios años. Los procesos de enseñanza en escuelas han enriquecido la creatividad, tanto de docentes como de estudiantes, que se han concentrado en el entendimiento técnico del recurso.

La educación no formal plantea un escenario distinto, muchas veces los espacios para su ejercicio no disponen de las garantías logísticas necesarias como pasa con la educación formal. El objetivo de este tipo de espacios educativos es la consolidación de comunidades de aprendizaje y sostenimiento preparados para enfrentar, en lo individual y colectivo, los problemas concretos de la vida. Como práctica social responde a un marco institucional que define el uso de ciertas herramientas o recursos con propósito claro: la restitución de derechos. En general, los recursos utilizados son muy básicos y su fortaleza radica en el carácter participativo de sus actividades.

Ahora bien, todas las actividades humanas están mediadas por el uso de herramientas, tanto materiales como simbólicas (Lion, 2006). Las primeras, son aquellos instrumentos que facilitan la realización de una actividad aplicando el menor esfuerzo; y las herramientas simbólicas son el conjunto de tradiciones, definiciones y leyes que promueven una convivencia social. El uso de una herramienta tendrá sentido en el marco de significación de una práctica social. Si el contexto de significación provisto, en la educación, no formal se concentra principalmente en el desarrollo de competencias para la vida, es posible usar a la robótica como herramienta mediadora, que aporte procesos que permitan cuestionar fenómenos propios de las relaciones humanas, como la identidad, el trabajo en equipo, la comunicación. Es decir, en el proceso de construcción de un robot se puede articular los contenidos simbólicos dispuestos en el tejido de significados de una cultura (Coll, 2008). En este ejercicio, lo importante es no perder de vista que la herramienta en sí no define al proceso formativo, sino es la práctica social quien otorga sentido a las actividades en donde se insertan las herramientas tecnológicas.

En cada momento histórico, las tecnologías han contribuido a promover metas colectivas, relaciones sociales, prácticas cotidianas y comportamientos diferentes. La importancia del uso de soportes tecnológicos en la educación no formal radica principalmente, en la posibilidad de interactuar de maneras diversas con los otros y con la información. Nutre el entorno de aprendizaje que mantiene vinculados a los individuos, por tanto, su valor en la generación de conocimiento dependerá de su aplicabilidad a situaciones concretas. Bajo esta lógica, la robótica no se define únicamente desde su valor instrumental (meramente herramientas para efectivizar un proceso), sino más bien por su uso simbólico, en asociación con situaciones complejas propias de la vida en sociedad. 
Luis Fernando Vallejos - Verónica Egas-Reyes - Diego Ordóñez-Camacho

La metáfora, recurso pedagógico en espacios de educación no formal mediados

por el uso de TIC: una experiencia ecuatoriana

\section{Aprendizaje por metáfora}

¿Qué es una metáfora? La Real Academia de la Lengua Española la define como la "traslación del sentido recto de una voz a otro figurado, en virtud de una comparación tácita". Es un tropo productor de sentido que facilita la comprensión valiéndose de la comparación para crear un universo de significados. Forma parte del lenguaje y está presente en todo el proceso de desarrollo del pensamiento, desde la temprana edad hasta la vejez, por tanto, está estrechamente ligada a los procesos de aprendizaje que hacen posible la abstracción del mundo (Parra-León y Marín-Díaz, 2017).

El uso de la metáfora implica dos procedimientos: el primero se refiere al traslado desde un sentido hacia otro figurado, y el segundo proceso da cuenta de cierto isomorfismo entre dos objetos o situaciones sin relación aparente. Esto quiere decir que la metáfora no tiene sentido único, por tanto, es un elemento fundamental del proceso de construcción de la subjetividad (Caballero, 2017).

Rodríguez (1988) señala que la metáfora es un recurso consistente pues hace uso de un objeto para crear una perspectiva nueva de otro. Refiere al proceso a través del cual el sentido y las relaciones de una teoría o un modelo son empleados para sugerir sentido o relaciones con otro modelo. La tarea comparativa de las metáforas involucra un proceso selectivo para visibilizar o invisibilizar elementos del fenómeno que se enuncia según el sentido que se desea producir, en consecuencia, la generación de significado se puede dirigir a la construcción de una realidad en específico. Como lo señalan Pochulú et al., (2011), para un proceso de aprendizaje es imperativo construir la realidad que se enuncia; la metáfora provee nuevos escenarios para pensar los fenómenos.

Al ser una herramienta simbólica es esencial para el proceso comunicativo, en consecuencia, tiene un valor educativo valioso, pues, además de facilitar la construcción de modelos de representación de la realidad, permite una interacción con los demás; por tanto, como señala Rodríguez (1988), se crean diversos modelos comportamentales a partir de las metáforas que se elaboran de la cultura.

Muchos de los saberes disciplinares son producto del conjunto de múltiples metáforas. En términos de educación no formal, cuando hablamos de aprendizaje por metáfora, nos referimos a un proceso cognitivo y creativo en el cual se produce sentido (aprendizajes) a partir de imágenes, conceptos o vivencias discutidas en los procesos de construcción de un proyecto tecnológico en asociación con cualquier otra circunstancia de la vida del participante. Por tanto, el aprendizaje por metáfora es un proceso pedagógico que:

- Facilita la comprensión de objetos o experiencias prácticas.

- Permite asociar eventos aparentemente inconexos.

- Aporta a la construcción de los contextos de significación.

- Al incidir sobre la subjetividad pude determinar modos de actuación.

- Evoca sentido y construye nuevas realidades.

- Refleja el universo de ideas con las que un colectivo concibe un fenómeno en un momento dado.

La metáfora constituye un elemento nuclear de pensamiento simbólico; canalizar su lógica hacia un quehacer pedagógico no es un trabajo complejo, pero sí demanda de construcciones discursivas claras en relación a los marcos de sentido donde se pretenda instalarla. 
Luis Fernando Vallejos - Verónica Egas-Reyes - Diego Ordóñez-Camacho La metáfora, recurso pedagógico en espacios de educación no formal mediados por el uso de TIC: una experiencia ecuatoriana

Dentro de los procesos de aprendizaje encontramos a la propuesta de Carlos Scolari (2015), quien a través de la Teoría de la Comunicación propone el uso de la metáfora, en las tecnologías, como herramienta de aprendizaje:

Las metáforas son fundamentales para entender el mundo que nos rodea y, además, ocupan un papel central en nuestra concepción de las tecnologías. Pero las metáforas no solo son importantes en nuestras conversaciones cotidianas: también desempeñan un papel fundamental en el discurso científico. Muchos nuevos paradigmas o modelos teóricos nacieron o se representan a través de metáforas. Estos recursos retóricos son muy útiles para dar sentido a los nuevos fenómenos que de otro modo serían casi imposibles de interpretar. Las metáforas generan categorías, organizan procesos y establecen oposiciones y jerarquías (Scolari, 2015, p. 27).

\section{Formación en competencias para la vida con el uso de la robótica}

El uso de la robótica como medio de enseñanza-aprendizaje, ha sido ampliamente utilizado en los entornos de educación formal. Los retos pedagógicos que demanda su implementación han podido ser atendidos por el sistema educativo tradicional en algunos países europeos. La robótica educativa, en la actualidad, es una excelente estrategia didáctica complementada con el apoyo de las tecnologías digitales (Vásquez, 2012). Se propone como enfoque didáctico que ofrece funcionalidad y aplicabilidad a los jóvenes, y se encuentra ubicada dentro de las llamadas competencias digitales, puesto que en el momento de resolver un problema o elaborar un producto específico, se potencian las habilidades intelectuales y se promueve la responsabilidad personal y de equipo, como también se potencia el uso transversal y contextualizado de contenidos.

La articulación entre la robótica y las competencias ha sido también investigada. Vásquez (2012) trabajó sobre la simulación robótica en el desarrollo de competencias básicas entre varias las materias curriculares, analizando las apreciaciones de docentes y alumnos sobre la viabilidad de la simulación robótica a través del trabajo por proyectos. Los resultados mostraron una alta apreciación de alumnado y profesorado sobre el proyecto y su funcionalidad en el desarrollo transversal e interdisciplinar de las competencias investigadas.

Encontramos también al programa educativo Castilla ejecutado el año 2013 (Cuartielles, 2014) que incorporó el uso de la plataforma Arduino en el sistema educativo español. En el proyecto participaron 400 estudiantes y 19 profesores de 23 institutos de Castilla de la Mancha. Gracias a esta experiencia se han derivado plataformas virtuales de enseñanza de programación con Arduino dentro de las aulas.

En Ecuador, Fundación Telefónica ha intentado emular esta experiencia haciendo uso de lo aprendido en el programa Castilla para llevar el uso de tecnología creativa (Arduino) dentro y fuera de las aulas. Este texto recoge algunas experiencias del proyecto Adolescentes Innovadores de Fundación Telefónica-IPC que utilizó recursos asociados a la programación y electrónica de la plataforma Arduino en los procesos de formación de competencias para la vida para adolescentes en situación de vulnerabilidad.

Lo primero que se descubre es que las plataformas de enseñanza de robótica diseñadas para la educación formal europea no tienen el mismo efecto en la educación no formal latinoamericana. Como lo advierte Lion (2006, p. 156), "no toda tecnología sirve a cualquier sociedad, ni toda sociedad puede absorber cualquier tecnología". Existen elementos de orden cultural, social o económico que imposibilitan 
su acceso. Los contextos educativos son diferentes y el marco de significación distinto, por ende, es necesario crear una metodología cuya pedagogía sea afín al entorno educativo no formal latinoamericano. Como salida se plantea el uso del aprendizaje por metáfora, estrategia que se suma al conjunto de actividades formativas para dar sentido al uso de los recursos tecnológicos.

Este proceso demanda un trabajo colaborativo para la construcción del conocimiento, lo cual implica tejer una red de cooperación y compromiso para el desarrollo de un proyecto determinado. Jenkins (2006) habla acerca de la cultura de la convergencia y postula que la misma no se asienta básicamente en una revolución tecnológica sino más bien en un fenómeno cultural que se apoya en la presencia de los consumidores/usuarios en una dinámica social, y muestra como ejemplo a: The Matrix, American Idol o Survivor. Él señala cómo la cultura participativa va junto con la fama, el éxito y la exposición masiva.

Es un ejercicio que solicita, en primera instancia, una observación profunda del entorno para identificar aquellos procesos que son parte de la cotidianidad de la comunidad u objetos pertenecientes a su simbología. Estos elementos servirán en lo posterior para hacer uso metafórico de los mismos con el propósito de introducir nuevos contenidos. En segundo lugar, este proceso merece un trabajo creativo para construir recursos o plantear actividades que posibiliten la asociación del contenido a introducir con la experiencia de la población en cuanto a los objetos o actividades que les sean más familiares. Como lo señala Coll (2008, p. 56), "las relaciones entre los sujetos y los objetos a través de herramientas no se dan mediante acciones solitarias, sino en un contexto de relaciones comunitarias, donde los cambios en las normas o en las formas de división de trabajo transforman radicalmente la actividad, por tanto, al sujeto".

A continuación, en la Sección 2 se amplían aspectos del aprendizaje en entornos educativos no formales. En la Sección 3 se traza un marco contextual para el uso educativo de nuevas tecnologías. En la Sección 4 se amplía en el impacto que puede tener la robótica en las experiencias de enseñanza-aprendizaje en entornos no formales y como competencia técnica dentro del marco de las competencias; posteriormente, en la Sección 5 se proponen conceptos sobre el aprendizaje por metáfora y su instrumentalización en ámbitos educativos. En la Sección 6 se expone el método enseñanza-aprendizaje por metáfora para entornos educativos no formales que dan sentido al uso de la robótica. En la Sección 7 se detalla la metodología utilizada para abordar el problema. Dentro de la Sección 8 se analizan los resultados, y, en la Sección 9 se presentan las conclusiones del caso.

\section{METODOLOGÍA}

Tabla 1

Zonas de intervención

\begin{tabular}{lll}
\hline Provincia & Cantones & Distribución \\
\hline Azuay & Chordeleg, Gualaceo & $22 \%$ \\
Imbabura & lbarra, Andrade Marín & $19 \%$ \\
Esmeraldas & Esmeraldas, Atacames, Muisne & $31 \%$ \\
El Oro & Santa Rosa, Arenillas, 28\% & \\
& Huaquillas & \\
\hline
\end{tabular}

El método de abordaje se construyó en el marco de trabajo del Proyecto Adolescentes Innovadores, que se desarrolló desde el mes de agosto del año 2013 hasta junio del 2016. Esta iniciativa fue el resultado de una alianza estratégica entre el 
Ministerio de Trabajo, la Fundación Telefónica y la Fundación IPC. Su objetivo consistió en crear espacios de educación no formal basados en el uso de recursos tecnológicos, que sirviesen para la prevención y disminución del trabajo infantil y adolescente desprotegido en las provincias de Azuay, Esmeraldas, El Oro e Imbabura.

Con este propósito, Adolescentes Innovadores construye una metodología que integra herramientas lúdicas y tecnológicas, enfocadas al desarrollo de competencias que aporten con aprendizajes prácticos canalizables a la construcción de un plan de vida. Con ello, se intenta que los participantes se mantengan dentro de la educación formal y continúen su proceso hasta una educación mínimo de tercer nivel. En la propuesta participan 2410 adolescentes entre los 12 y 17 años de edad, en situación de vulnerabilidad: el $80 \%$ se encuentra en riesgo de trabajo infantil y el $20 \%$ son población infantil trabajadora, todos residentes en zonas urbano-marginales de los cuales el $59.10 \%$ son hombres y el $49.90 \%$ mujeres. El $78 \%$ de la población es mestiza, el $13 \%$ afro ecuatoriana, el $5 \%$ se define como blanco y el $4 \%$ indígena.

La metodología que surge de esta experiencia se fundamenta en la práctica investigación-acción, sistema ampliamente desarrollado en el contexto de las ciencias sociales en Latinoamérica. Considera la participación activa de los actores sociales vinculados al fenómeno de estudio y en la creación de su propio método de trabajo, por tanto, las actividades son motivadas por sus necesidades e intereses. Como tal es un proceso dinámico que conjuga en simultáneo praxis y desarrollo teórico. Para esta práctica en particular, los métodos de intervención fueron el producto de aprendizajes provenientes del intercambio reflexivo entre los adolescentes participantes, coordinadores de la investigación, promotores y técnicos sociales, representantes institucionales (Gobiernos Autónomos Descentralizados, GAD, ONG, unidades educativas y pastorales) cuya sinergia direcciona al proyecto según las particularidades del contexto de intervención. Esta lógica de estudio se implantó en todo el proceso de construcción metodológica (Tabla 2), desde el acopio de datos, el procesamiento de información, el análisis de resultados y las conclusiones, cada una alimentada por señalamientos de los diferentes actores sociales.

El proceso investigativo duró dos años y seis meses, tiempo en el cual se desarrollaron cuatro etapas. El objetivo de las etapas 1 y 2 fue el diagnóstico en la etapa 3, de mayor duración, se construyó y ejecutó la propuesta metodológica bajo el enfoque investigación-acción y se analizaron los resultados. El objetivo de la etapa 4 fue socializar la metodología y transferir la población participante a instituciones sociales o programas de participación ciudadana en cada localidad. En cada etapa se crearon instrumentos especiales que permitieron el ordenamiento y las síntesis de los datos a través de análisis estadísticos básicos para la información cuantitativa (encuestas) y análisis de contenido para la información cualitativa (talleres y encuentro grupales).

En la etapa 1, se realizaron visitas de terrenos y entrevistas a personal de las áreas sociales pertenecientes a los GAD cantonales y parroquiales, quienes aportaron información contextual. En la etapa 2, se ejecutó el proceso de línea base por medio de fichas de encuentros e instrumentos relacionales. La etapa 3 se destinó a la creación y ejecución de los módulos de trabajo en los cuales se integraron tecnologías creativas. Finalmente, en la etapa 4 se cerraron las operaciones con la transferencia metodológica y poblacional a programas sociales de los GAD.

La metodología de intervención se desarrolló principalmente en las etapas 2 y 3; como resultado surgen los módulos de trabajo orientados a fortalecer características asociadas a las competencias liderazgo-responsabilidad, innovación-creatividad y plan de vida como se muestra en la Tabla 3. La estructura metodológica considera en principio que el trabajo profundo de las temáticas y su articulación producirán el 
fortalecimiento de la competencia designada en su conjunto. Para cada temática (módulo) se plantea el desarrollo de un microproyecto, que consiste en un reto práctico de uso tecnológico que los grupos de adolescentes deben cumplir. Los participantes son quienes proponen el tipo de artefacto tecnológico que desean construir usando la plataforma de hardware libre Arduino y otros elementos electrónicos.

Tabla 2.

Proceso de desarrollo metodológico

\begin{tabular}{|c|c|}
\hline \multirow[t]{2}{*}{ Diagnóstico } & $\begin{array}{l}\text { Etapa } 1 \text { Preparación previa de proyecto: Observaciones en } \\
\text { territorio y entrevistas con responsables locales de proyectos } \\
\text { sociales. Se recopila información local sobre la situación de los } \\
\text { adolescentes, de su familia y de la comunidad. Se desarrollan los } \\
\text { primeros contactos inter institucionales de cooperación. }\end{array}$ \\
\hline & $\begin{array}{l}\text { Etapa } 2 \text { Desarrollo de línea base: Esta etapa comprendió el diseño } \\
\text { y montaje de las herramientas para el levantamiento de línea base y } \\
\text { el análisis de datos. Se construyen dos tipos de instrumentos, una } \\
\text { ficha de encuesta y una herramienta de interacción. Con la } \\
\text { información integrada se construye el esquema metodológico y la } \\
\text { estructura técnica de trabajo con la cual se desarrollará la propuesta. }\end{array}$ \\
\hline $\begin{array}{l}\text { Desarrollos } \\
\text { de la } \\
\text { propuesta }\end{array}$ & $\begin{array}{l}\text { Etapa } 3 \text { Metodología de intervención: Se desarrollan los módulos } \\
\text { de trabajo referentes a Liderazgo-responsabilidad, creatividad e } \\
\text { innovación y plan de vida. Al finalizar esta etapa se realiza } \\
\text { nuevamente la ficha de encuesta y su datos son estudiados } \\
\text { comparativamente con los obtenidos en las fichas de encuenta de } \\
\text { etapa } 2 \text { para evaluar resultados. }\end{array}$ \\
\hline $\begin{array}{l}\text { Cierre de } \\
\text { proceso }\end{array}$ & $\begin{array}{l}\text { Etapa } 4 \text { Cierre de procesos-traspaso de población a } \\
\text { instituciones aliadas: Una vez culminado el proceso formativo, se } \\
\text { prosigue con el traspaso de la población y la metodología a las } \\
\text { instituciones públicas o privadas abiertas en dar continuidad al } \\
\text { trabajo de acompañamiento con los adolescentes. En este ciclo, los } \\
\text { equipos de técnicos sociales del proyecto cierran sus operaciones y } \\
\text { se capacita a los técnicos de las instituciones aliadas. }\end{array}$ \\
\hline
\end{tabular}

Tabla 3.

Estructura metodológica

\begin{tabular}{lll}
\hline Liderazgo & y $\begin{array}{l}\text { Creatividad e innovación } \\
\text { responsabilidad }\end{array}$ & $\begin{array}{l}\text { Plan de vida } \\
2015\end{array}$ \\
\hline
\end{tabular}

2014

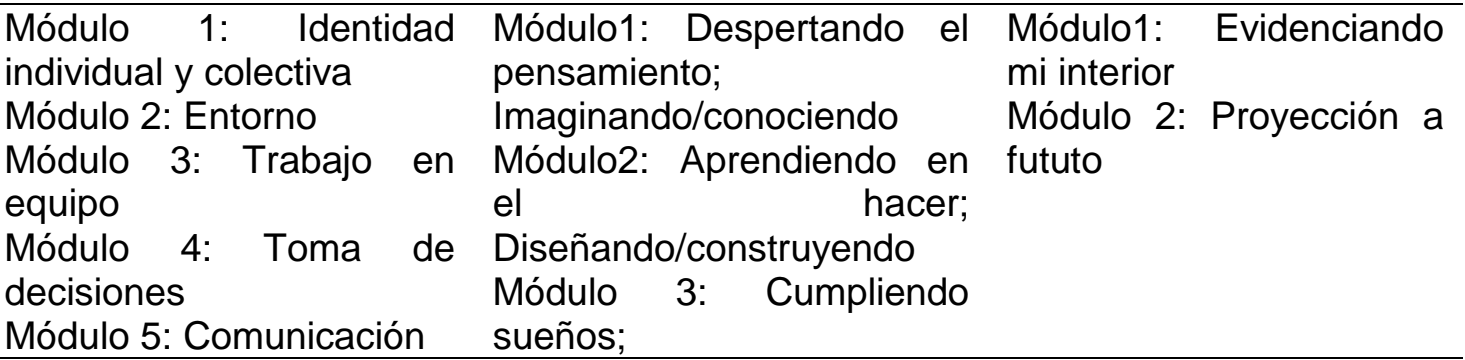


Luis Fernando Vallejos - Verónica Egas-Reyes - Diego Ordóñez-Camacho La metáfora, recurso pedagógico en espacios de educación no formal mediados por el uso de TIC: una experiencia ecuatoriana

Módulo 6: $\quad$ Expresión Transformando/Inventando individual y colectiva

Durante el proceso Adolescentes Innovadores, los productos realizados en los microproyectos variaron desde robots con materiales reciclados, parques de diversiones y ciudades (maquetas) digitales. Estos artefactos, además de ser evidencian física del proceso técnico, contienen una función simbólica al actuar como medio para el pronunciamiento de los adolescentes quienes pueden transmitir lo que sienten interiormente, con respecto a su mundo relacional, o por su comunidad a través del objeto que ellos han fabricado. En este sentido se configura el conjunto de actividades formativas que guían esta práctica social; las actividades son diseñadas en la medida en que sean culturalmente relevantes para los adolescentes y su entorno social.

El microproyecto es la parte nodular de la propuesta metodológica; en su desarrollo convergen actividades lúdicas, aprendizaje por metáfora, contenidos específicos de las temáticas de cada competencia y el uso de recursos tecnológicos. Todo ello se organiza en un esquema previamente planificado y sostenido con un marco teórico de fondo.

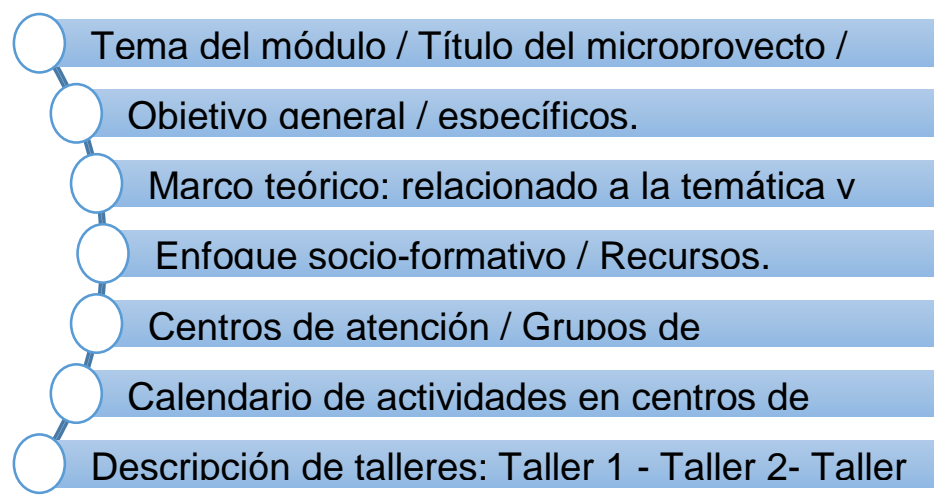

Figura 1. Elementos del microproyecto

Los microproyectos se formulan para tratar la temática de una competencia determinada de la estructura metodológica y elaborar un producto tecnológico. En la Figura 1 se exponen los elementos que conforman un microproyecto

Conscientes de que la información a recolectar en el proceso de línea base podría ser sensible para el adolescente, se diseñaron instrumentos de investigación que facilitaron el contacto con los participantes permitiéndolos expresarse libremente. 


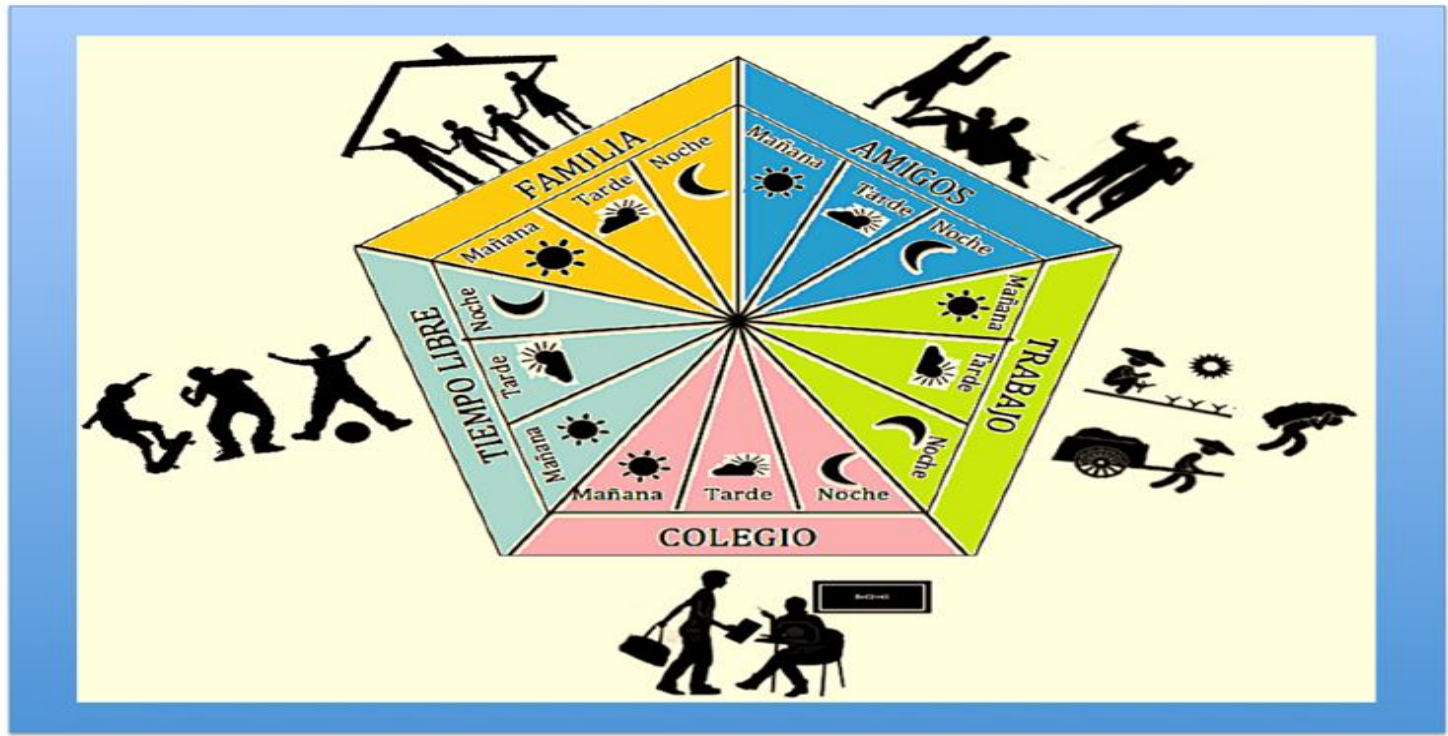

Figura 2. Ficha lúdica de encuesta

Uno de los principales instrumentos fue una ficha de encuesta, la cual se diseñó para proporcionar datos sobre cinco aspectos de la vida del adolescente: familia, situación de uso del tiempo libre, acceso a la tecnología, educación y condiciones del trabajo infantil. Lo que se propuso fue el juego de una ruleta, la cual estaba dividida en cinco segmentos, correspondientes cada uno de los aspectos de la ficha de encuesta que se pretendía indaga, como lo muestra.

En esta dinámica, la experiencia de ejecutar una encuesta se convirtió en una oportunidad de relacionamiento más cercana y menos invasiva. La herramienta de interacción es un recurso lúdico para generar una relación de confianza entre el entrevistador y el entrevistado, la cual facilite el fluir de la conversación, haciendo de la entrevista un proceso de intercambio amigable.

\section{RESULTADOS}

Los resultados que se muestran a continuación fueron obtenidos directamente del grupo de adolescentes que participaron en el proceso formativo "Adolescentes innovadores". Los indicadores cuantitativos y cualitativos provienen del procesamiento de una encuesta realizada en el año 2015, a 1274 adolescentes que participaron de manera continua en el proceso instalado en las provincias de Azuay, El Oro, Esmeraldas e Imbabura.

Como parte de la medición de impacto se intentará relacionar la importancia que tiene el proceso pedagógico del aprendizaje por metáfora para el uso de la tecnología en entornos de aprendizaje no formal. Se mostrará el resultado de esta iniciativa en su objetivo de contribuir con la prevención y disminución del trabajo infantil y adolescente desprotegido, así como también su relación de impacto con la reinserción del adolescente en condición de vulnerabilidad al sistema de educación formal.

El uso de recursos tecnológicos (robótica) tiene relevancia dentro del contexto de significación creado por la práctica social. En el proceso "Adolescentes innovadores" la electrónica y robótica debía aportar al desarrollo de competencias para la vida, es decir, proveer aprendizajes concretos aplicables a los problemas cotidianos. Si se apela al valor instrumental del recurso tecnológico no pasará de ser una herramienta 
Luis Fernando Vallejos - Verónica Egas-Reyes - Diego Ordóñez-Camacho

La metáfora, recurso pedagógico en espacios de educación no formal mediados por el uso de TIC: una experiencia ecuatoriana

técnica, como lo es un martillo o un serrucho. Como se observa en la Figura 3, que ilustra las preferencias de los adolescentes en los espacios de formación, solo el $5.10 \%$ de los adolescentes disfruta de construir robots y el $4.08 \%$ de la electrónica como actividades aisladas.

El valor simbólico que determina el uso y apropiación de los recursos tecnológicos depende del conjunto integrado de otras actividades que dan sentido al proceso de elaboración de un producto. Este contexto de significación es construido a través del aprendizaje por metáfora, proceso que permite relacionar la experiencia de producir un artefacto tecnológico con las dificultades asociadas a retos de la vida como la toma de decisiones, la construcción de la identidad, entre otras muchas temáticas. Volviendo a la Figura 3, observamos que $23.63 \%$ de los adolescentes disfrutan de las actividades en todo su holística, es decir, encuentran sentido al proceso formativo en gran medida porque encuentra articuladas las actividades de carácter tecnológico con los procesos de vida de un adolescente. Esta relación es amalgamada por el efecto del recurso lúdico.

El resultado del proyecto "Adolescentes innovadores" en relación al objetivo de aminorar los indicadores de deserción escolar y apoyar la reinserción de los adolescentes en situación vulnerable en la educación formal, indica que, a finales del 2015 , el $96 \%$ de los participantes se mantuvieron en el sistema educativo, el $3.92 \%$ prefirieron abstenerse de responder y el $0.08 \%$ solamente abandonó los estudios. La propuesta formativa a través del uso de recursos tecnológicos posibilita el aparecimiento de grupos de adolescentes, amigos que encuentran similitudes en sus intereses y condiciones de vida y que finalmente se sostienen entre sí para enfrentar las contingencias de su desarrollo.

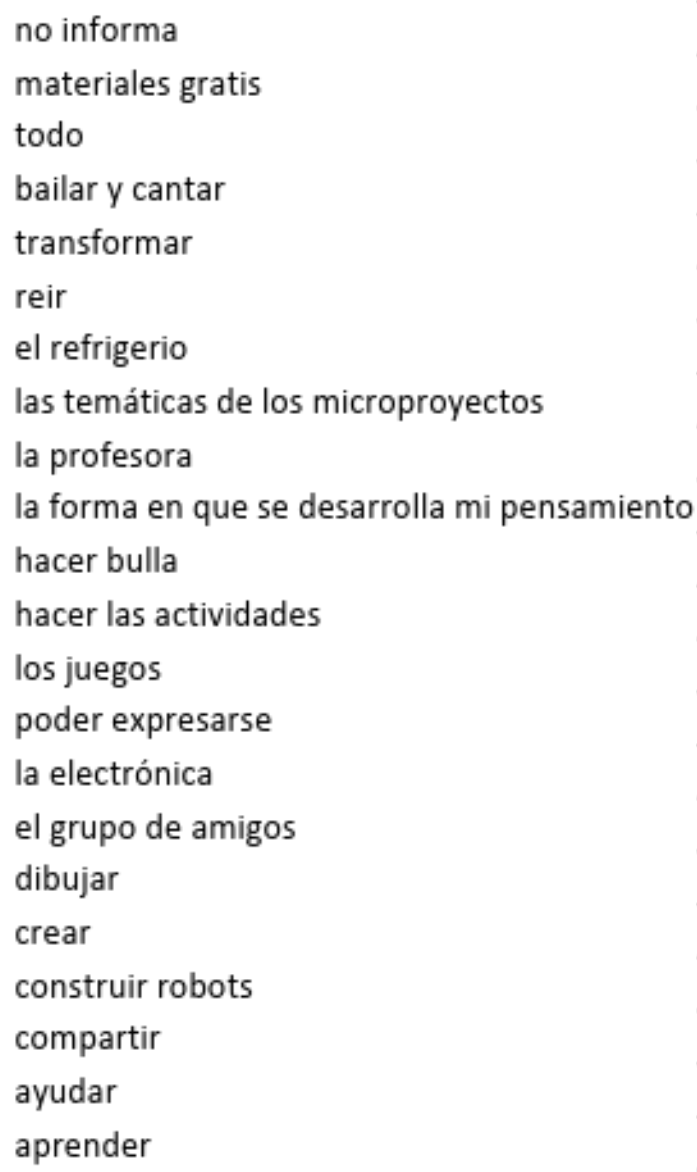

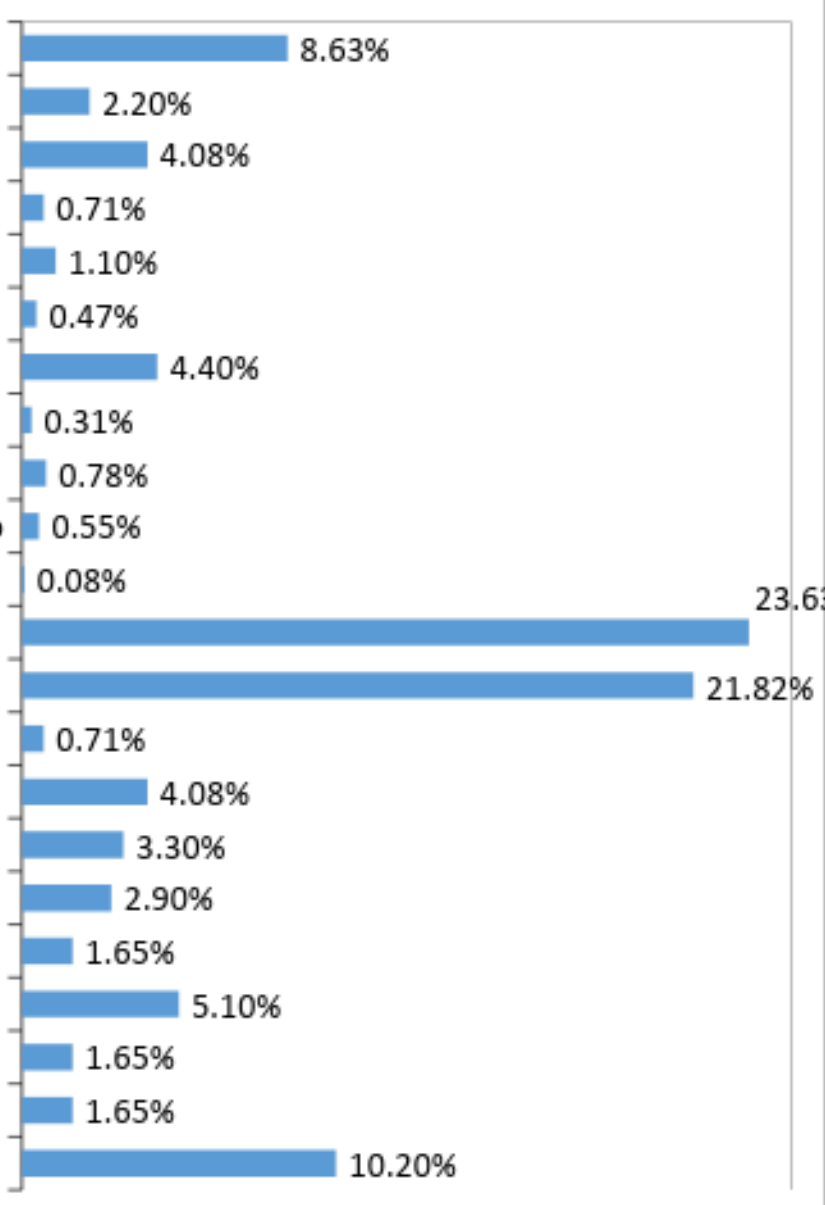


Figura 3. Lo que más le gusta al adolescente del proyecto

Las causas que empujan a un niño o un adolescente hacia el trabajo son muy variadas, se reconocen fuertes atavismos culturales que significan a la actividad laboral como proceso formativo. Sin embargo, es fundamental entender a este fenómeno social desde la particularidad de cada caso, dado que los significantes que promueven la actividad laboral pueden comprometer el mundo emocional y psíquico del adolescente. La práctica del proyecto "Adolescentes innovadores" logró que el $57.46 \%$ de los adolescentes abandonen el trabajo y se concentren en actividades formativas. El $38.38 \%$ de participantes combina estudios con actividades laborales y solo el $4.16 \%$ se dedica netamente a labores informales.

El efecto cualitativo de este proceso formativo que apostó al uso de la tecnología y herramientas pedagógicas como recurso para el descubrimiento de habilidades canalizables a un plan de vida, puede visualizarse en las consideraciones que presenta el adolescente, cuando se refiere a sus prioridades actuales. En la Figura 4, observamos la respuesta de los adolescentes cuando se les pregunta sobre lo que creen que es necesario para alcanzar una meta. Notamos que el $53.69 \%$ identifica que el estudio es necesario para sus proyectos de vida, reconocen, además, la importancia de fijar su voluntad y contar con el apoyo familiar en este camino. Esta es una referencia cualitativa que demuestra la complementariedad que existe entre la educación no formal, la educación formal y la educación informal.

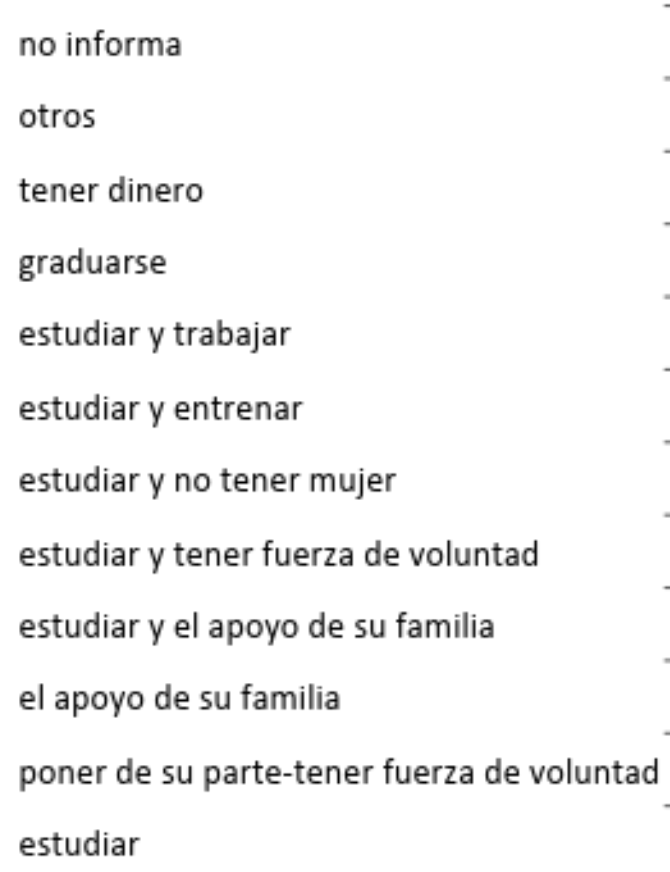

Figura 4. ¿Qué necesita el adolescente para llegar a su meta?

Los encuentros con los adolescentes se mostraron como espacios de interacción a ser vividos como una experiencia significativa, como espacios para el cuestionamiento, el intercambio de perspectivas, la construcción colectiva de saberes, el momento ideal para la articulación entre aprendizajes y subjetividad. Las vivencias son la materia prima para el desarrollo de competencias para la vida; al respecto, Perrenoud $P$. sugiere: "Una competencia no se define a partir de saberes predeterminados. Debería ser al revés, partir de las situaciones y prácticas sociales más comunes, inferir de ellas unas competencias e identificar los saberes, habilidades y actitudes que dichas 
competencias requieren como recursos" (2012, p. 34). El sentido que define el uso de los recursos tecnológicos es producto de las metáforas propuestas. Dichas asociaciones cumplen un doble propósito: en principio ilustran el funcionamiento técnico del artefacto tecnológico y en segundo lugar hacen del objeto una herramienta simbólica para significar una circunstancia de vida.

A continuación, describiremos un ejemplo que ilustra este señalamiento.

\section{Ejemplo}

En uno de los encuentros relacionados al módulo "toma de decisiones" realizados en la parroquia Simón Bolívar de Gualaceo, en la provincia de Azuay, se requiere explicar la diferencia entre la lógica binaria y lógica analógica, procedimiento necesario para entender el funcionamiento de la placa arduino, e identificar los pines para la construcción de un robot. Como actividad de mediación para el aprendizaje por metáfora se propone un juego. El mismo consiste en dirigir una serie de preguntas referentes a la vida del adolescente en su comunidad, ante las cuales, el participante puede contestar con cualquier otra palabra excepto: "sí o no".

La actividad comienza, el promotor dirige sus preguntas hacia cada adolescente del grupo quienes expectantes contestan sobre los lugares preferidos de su barrio, las actividades que hacen sus amigos, los sitios donde se reúnen, la relación con los profesores de colegio, etcétera. Aunque la mayoría de los adolescentes fracasa en su intento de evitar utilizar el "sí" y el "no" como respuestas a las preguntas cerradas, la actividad genera una relación de confianza entre el técnico social y el grupo que participa colaborativamente.

Una vez terminada la actividad se asocia el juego, en principio, con el funcionamiento de la lógica binaria, cuyo símil se representa en las respuestas de si y no, que en electrónica se traduce con: alto-bajo o 0v-5v. A continuación, se explica el funcionamiento de la lógica analógica que refiere al rango de posibilidades cuyas respuestas en el juego variaron entre: tal vez, a veces, quien sabe, recursos que utilizaron para evitar el 'sí' o el 'no'. En electrónica, este conjunto de alternativas representa a todos los rangos (1024 niveles) de voltaje intermedios entre 0v-5v. A su vez, esta experiencia lúdica mostró que hay ciertas circunstancias en la vida en las cuales decidimos de manera radical con un 'sí' o un 'no', y en otras situaciones preferimos inclinarnos por los matices. Esto que sucede en la lógica binaria y analógica también puede pasar en la vida como escenario, por tanto, es importante reconocer las intenciones que nos empujan a una u otra decisión.

La metáfora tiene efectos en distintas dimensiones como el lenguaje, los conceptos, en la realidad que enuncia y en la acción de los sujetos. El contexto de significación del entorno de educación no formal pretenderá relacionar el uso de un recurso tecnológico con alguna situación concreta de la vida. La metáfora actuante puede ser un proceso, objeto o vivencia por donde se canalicen estos saberes.

\section{CONCLUSIONES}

Construir una metodología para una educación no formal que haga uso de recursos tecnológicos como herramienta para el autoconocimiento, demanda el uso de estrategias pedagógicas que permitan asociar el aprendizaje técnico con las dificultades que atraviesa una persona en su diario vivir. El uso de la tecnología en este campo rebasa lo instrumental; no se limita únicamente a que los participantes aprendan robótica o programación. Más bien se reconoce una relación dialéctica de modificación mutua entre el objeto tecnológico y la persona que hace uso del mismo. La estrategia pedagógica que eslabona esta relación es la metáfora, mecanismo cognitivo elemental para conocer el mundo; constituye un elemento nuclear de 
Luis Fernando Vallejos - Verónica Egas-Reyes - Diego Ordóñez-Camacho

La metáfora, recurso pedagógico en espacios de educación no formal mediados por el uso de TIC: una experiencia ecuatoriana

pensamiento simbólico que posibilita la construcción del sentido y el tejido de nuevas cadenas semánticas. Esto posibilitó en los adolescentes adquirir dominio y responsabilidad sobre las circunstancias, combinando saberes con actitudes en una acción común. Al mismo tiempo, la intervención permitió reducir la situación social de riesgo con relación al trabajo infantil y un acercamiento de la población a actividades formativas.

El objetivo de la metodología no es enseñar electrónica a los adolescentes, esto llega por añadidura, sino proveer experiencias acordes a su realidad y a la de su medio. Nutrir su experiencia de vida con circunstancias de experimentación en las que participen sus pares, aportar a su autoconocimiento personal y su capacidad crítica; en este sentido se define el uso y apropiación del recurso tecnológico, en la medida en que se haga uso del objeto, que este sirva para significar los sucesos de vida y contribuya en la formación subjetiva e identitaria del adolescente.

\section{AGRADECIMIENTOS}

Agradecemos al Ministerio de Trabajo del Ecuador, a la Fundación TelefónicaEcuador y a la Fundación IPC. Asimismo, la colaboración interinstitucional requerida para presentar este trabajo fue posible gracias al Proyecto de Investigación para el Desarrollo (PRD-ARES/CCD) Bélgica-Ecuador "Renforcement des espaces de médiation communautaire des jeunes dans les situations de violence et de souffrance psychosociales".

\section{BIBLIOGRAFÍA}

Aberastury, A. y Knobel, M. (2004). La adolescencia normal: un enfoque psicoanalítico. Paidos.

Belçaguy, M., Cimas, M., Cryan, G. y Loureiro, H. (2015). Adolescencia y tecnologías de la información y la comunicación. Ficha de Cátedra I, Psicología Evolutiva: Adolescencia. Prof. a cargo Dr. José Barrionuevo. Oficina de Publicaciones. Universidad de Buenos Aires.

Bunch, G. (2016). Claves para una educación inclusiva exitosa: Una mirada desde la experiencia práctica. Revista de educación inclusiva, 1(1).

Caballero, A. (2017). La importancia del componente metafórico en el aprendizaje de E/2L. Acercamiento pragmático. redELE: Revista Electrónica de Didáctica ELE, (29), 7.

Castells, M. y Andrade, J. (2010). La sociedad red: una visión global. Enl@ce, 7(1).

Castro Pérez, M.,y Morales Ramírez, M. E. (2015). Los ambientes de aula que promueven el aprendizaje, desde la perspectiva de los niños y niñas escolares. Revista Electrónica Educare, 19(3). Recuperado a partir de http://www.redalyc.org/resumen.oa?id=194140994008

Cobo, R., Moravec, J. (2011). Aprendizaje Invisible. Hacia una nueva ecología de la educación. Col-lecció Transmedia XXI. Laboratori de Mitjans Interactius / Publicacions i Edicions de la Universitat de Barcelona. Barcelona

Coll, C. (2008). Psicología de la educación virtual: aprender y enseñar con las tecnologías de la información y la comunicación. Ediciones Morata. 
Luis Fernando Vallejos - Verónica Egas-Reyes - Diego Ordóñez-Camacho

La metáfora, recurso pedagógico en espacios de educación no formal mediados por el uso de TIC: una experiencia ecuatoriana

Correa, A. (2017). La práctica del aprendizaje cooperativo. Madrid: Narcea de Ediciones. 160 págs. ISBN: 978-84-277- 2316-0. Revista de Investigación en Educación, 15(1), 85-86.

Costa, S., Cuzzocrea, F. y Nuzzaci, A. (2014). Usos de internet en contextos educativos informales: Implicaciones para la educación formal. Comunicar, 21(43).

Cuartielles, D. (2014). ARDUINO, herramienta para la educación en el entorno tecnológico. Presentado en Tercera Edición de Campus Party en Ecuador, Ecuador. Recuperado a partir de http://fundaciontelefonica.com.ec/conferencias/arduino-herramienta-para-laeducacion-en-el-entorno-tecnologico/

Esteve, F. (2016). Bolonia y las TIC: de la docencia 1.0 al aprendizaje 2.0. La Cuestión Universitaria, 0(5), 58-67.

Garganté, A. y Majós, T. (2006). La práctica psicopedagógica en educación no formal. Editorial UOC.

Grasso, M., Pagola, L.,y Zanotti, A. (2017). Políticas de inclusión digital en Argentina. Usos y apropiaciones dentro y fuera de la escuela. Pixel-Bit. Revista de Medios y Educación, (50).

Jenkins, Henry. (2006). Convergence culture.Where old and new media collide. Nueva York: University Press.

Lion, C. (2006). Imaginar con tecnologías: relaciones entre tecnologías y conocimiento. Editorial Stella.

Martínez, M., Sádaba, C. y Serrano Puche, J. (2018). Desarrollo de competencias digitales en comunidades virtuales: un análisis de "ScolarTIC". Revista Prisma Social, 0(20), 129-159. Recuperado de http://revistaprismasocial.es/article/view/2318

Martínez, G., Nolla, N., Vidal, M. y De la Torre Navarro, L. M. (2016). Los entornos personales de aprendizaje en los procesos de formación formales e informales. Educación Médica Superior, 30(3), 599-608.

Medina, M. (2015). Influencia de la interacción alumno-docente en el proceso enseñanza-aprendizaje. PAAKAT: Revista de Tecnología y Sociedad, O(8). Recuperado a partir de http://www.udgvirtual.udg.mx/paakat/index.php/paakat/article/view/230

Observatorio Social del Ecuador. (2004). La Niñez y Adolescencia en el Ecuador Contemporáneo. Avances y Brechas en el Ejercicio de Derechos. Unicef. Recuperado a partir de https://www.unicef.org/ecuador/NA_Ecuador_Contemporaneo.pdf

Palma, D., Agudelo, C. y Aguilar, P. E. C. (2017). Aprendizaje significativo: opción pedagógica constructivista en educación básica colombiana. Gestión, competitividad e innovación, 5(2). Recuperado a partir de https://pca.edu.co/investigacion/revistas/index.php/gci/article/view/115

Parra-León, G.,y Marín-Díaz, D. (2017). De la metáfora del perfeccionamiento humano al aprendizaje permanente: el desdoblamiento del concepto de educación. Cadernos de Educação, O(55). https://doi.org/10.15210/caduc.v0i55.10414

Perrenoud, P. (2012). Cuando la escuela pretende preparar para la vida: ¿desarrollar competencias o enseñar otros saberes? Grao. 
Luis Fernando Vallejos - Verónica Egas-Reyes - Diego Ordóñez-Camacho

La metáfora, recurso pedagógico en espacios de educación no formal mediados por el uso de TIC: una experiencia ecuatoriana

Pessoa, M., Serrano, M. y Rodríguez, J. (2015). Aprendizaje informal, alfabetización mediática e inclusión social. Descripción de una experiencia. Profesorado. Revista de Currículum y Formación de Profesorado, 19(2), 75-91.

Pochulú, M., Abrate, R.,y Visokolskis, S. (2011). La metáfora en la educación: Descripciones e implicaciones. Publicaciones Universitarias Argentinas.

Ramírez, M. (2017). La educación no formal. Reflexiones sobre una brillante alternativa. Metal, (3), 168-175.

Reig, D. (2012) Revolución social, cognitiva y creativa: desde las TIC, hacia las TAC y las TEP. Disponible en: http://www.dreig.eu/caparazon/2012/02/14/tepclavedelcambio/ y http://encuentro.educared.org/group/hacia-lasescuelas-3-0-ylosestudiantes-3-0/page/dolors-reig

Rodríguez, J. (1988). La metáfora en la enseñanza. Enseñanzay Teaching: Revista Interuniversitaria de Didáctica, 6.

Romero, M.,y Turpo Gebera, O. (2015). Serious Games para el desarrollo de las competencias del siglo XXI. Revista de EducacióN a Distancia, 0(34).

Recuperado de http://revistas.um.es/red/article/view/233511

Ruiz, P. y López, A. (2017). El estilo de aprendizaje y su relación con la educación entre pares. Revista de Investigación Educativa, 36(1), 221-237.

https://doi.org/10.6018/rie.36.1.233731

Scolari, C. 2015. Introducción: Ecología de los medios: de la metáfora a la teoría (y más allá). https://drive.google.com/file/d/0B19fnVg9hoDYy03V3NZVndGWlk/view

Sierra, F. (2013). Ciudadanía, tecnología y cultura. Editorial GEDISA.

Suárez, E. y Santana, L. (2016). La educación no formal: Una estrategia para la construcción de actores sociales. GénEros, 8(25), 13-17.

Valenzuela, M. (2016). Adquisición de valores por adolescentes en educación no formal. Variables predictoras y comparativa de programas (PhD Thesis). Universidad de Zaragoza.

Vázquez, E. (2012). Simulación robótica con herramientas 2.0 para el desarrollo de competencias básicas en eso. Un estudio de casos. Teoría de la Educación. Educación y Cultura en la Sociedad de la Información, 13 (2), 48-73.

Vega, A. (2016). A vueltas con la educación inclusiva: lo uno y lo diverso. Revista de Educación Inclusiva, 1(1).

Zapata-Ros, M. (2015). Teorías y modelos sobre el aprendizaje en entornos conectados y ubicuos. Bases para un nuevo modelo teórico a partir de una visión crítica del "conectivismo". Teoría de la Educación. Educación y Cultura en la Sociedad de la Información, 16(1). Recuperado a partir de http://www.redalyc.org/resumen.oa?id=201038761006 
Luis Fernando Vallejos - Verónica Egas-Reyes - Diego Ordóñez-Camacho La metáfora, recurso pedagógico en espacios de educación no formal mediados por el uso de TIC: una experiencia ecuatoriana

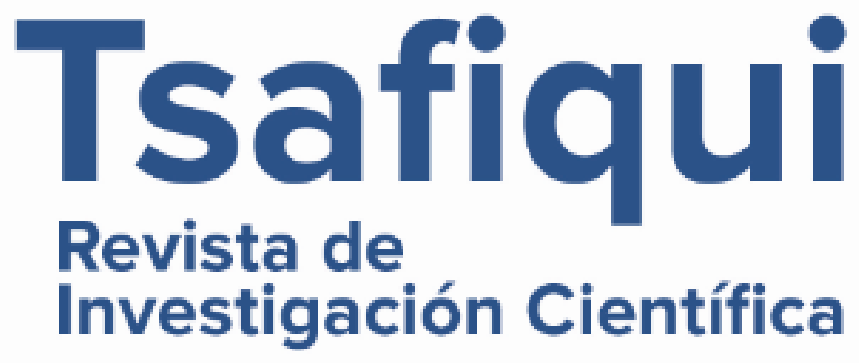

